\title{
Quantitative STEM Imaging and Multislice Simulation of Stacking Fault Defects for Exciton Trapping in GaAs
}

Steven Spurgeon ${ }^{1}$, Bethany Matthews ${ }^{1}$, Peter Sushko ${ }^{1}$, Xiayu Linpeng ${ }^{2}$, Maria Viitaniemi ${ }^{2}$, Mikhail Durnev $^{3}$, Mikhail Glazov ${ }^{3}$, Andreas Wieck ${ }^{4}$, Arne Ludwig ${ }^{4}$ and Kai-Mei Fu ${ }^{2}$

${ }^{1}$ Pacific Northwest National Laboratory, Richland, Washington, United States, ${ }^{2}$ University of Washington, Seattle, Washington, United States, ${ }^{3}$ Ioffe Institute, St. Petersburg, Saint Petersburg City, Russia, ${ }^{4}$ Ruhr-Universität Bochum, Bochum, Bremen, Germany

Control of defects in bulk semiconductors such as GaAs is essential to realize novel excitonic phases for emerging quantum devices. In these systems, stacking fault (SF) defects are common, leading to local variations in optical properties, but the exact configuration of such defects and their interaction with excitons are not fully understood. Here we describe the use of aberration-corrected scanning transmission electron microscopy (STEM) imaging to directly examine SF defects in the GaAs system. In particular, we quantify the structure and properties of several SF configurations through multislice image simulations based on ab initio calculations. We show that this approach can reveal significant differences in exciton trapping behavior and suggest ways to control quantum systems.

As shown in Figure 1.A, we considered two kinds of micron-sized defects, pyramidal and trapezoidal, in bulk GaAs [1]. These features can be detected through their unique optical emission properties as well as their morphology in the focused ion beam (FIB) microscope. Cross-sectional FIB lift outs were extracted from each type of defect and imaged using both low- and high-angle annular dark field (LAADF / HAADF) STEM, as shown in Figures 1.C-E. The former technique is more sensitive to strain fields around the defects and can be used to highlight their position in the sample, while the latter can directly resolve their atomistic structure. To improve matching to theory models, we collected a series of 10 frames at 1024 x 1024 px sampling and $2 \mu \mathrm{s} \mathrm{px}^{-1}$ dwell time; these were subsequently non-rigid aligned and template matched using the SmartAlign plugin [2]. The resulting images, shown in Figure 2, were then used as the basis to build and refine atomistic models. Importantly, we observe that the two bulk defects consist of either single extrinsic (for the pyramidal defect shown in Figure 2.A) or extrinsic-intrinsic SF pairs (for the trapezoidal defect shown in Figure 2.B). A series of multislice image simulations were performed using the PRISM code [3] to correlate ab initio calculations to the experiment, as shown in Figure 2.A. Our theory calculations show that a substantial built-in electric field is present in these materials and that the double SF structure can lead to nearly two-fold increase in exciton dipole moment, with significant implications for exciton trapping behavior. 


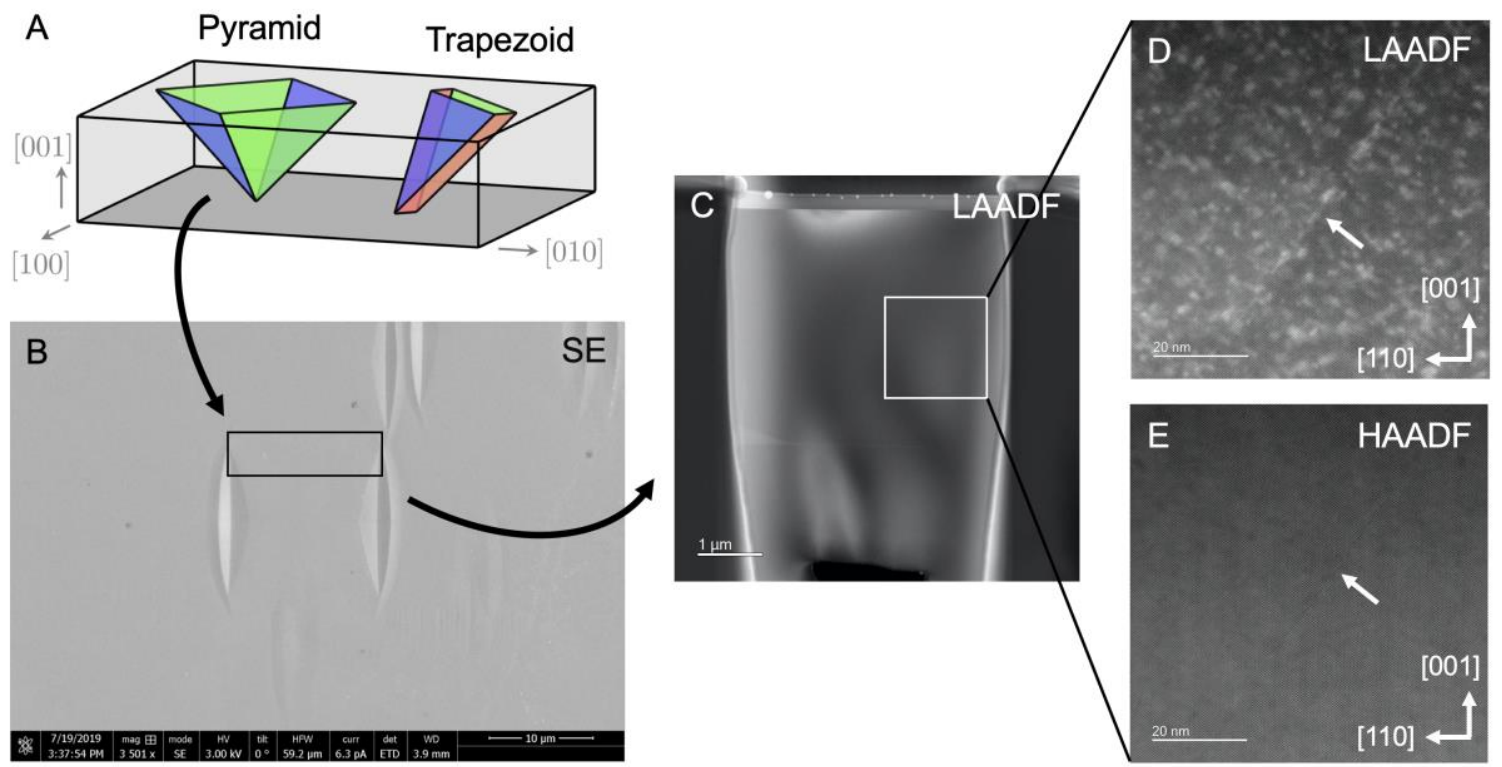

Figure 1. (A) Illustration of stacking fault defect configuration in GaAs. (B) Secondary electron (SE) image showing the pyramidal region from which a FIB lift out is extracted. (C-D) Cross-sectional STEMLAADF imaging highlighting the presence of a SF defect. (E) Cross-sectional STEM-HAADF image showing the atomistic configuration of the SF in the pyramidal defect.

\section{Pyramid}

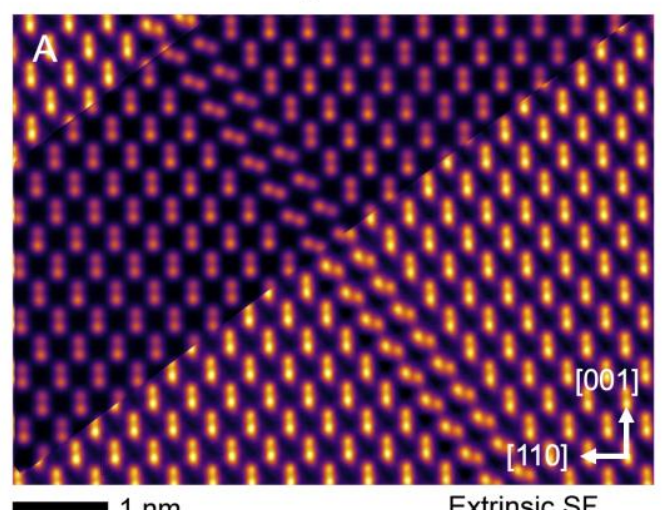

$1 \mathrm{~nm}$
Extrinsic SF

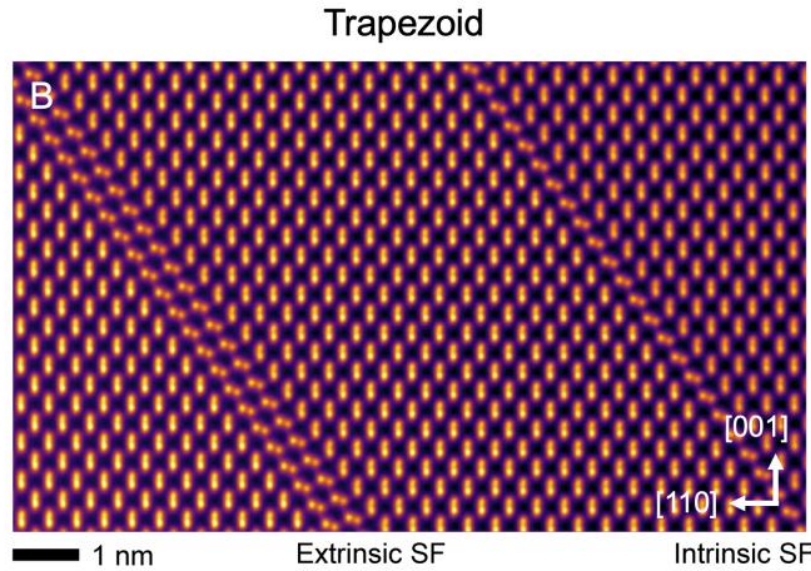

Figure 2. Drift-corrected and template-matched STEM-HAADF images of the pyramidal (A) and trapezoidal (B) defect configurations, which consist of a single extrinsic SF and an extrinsic-intrinsic SF pair, respectively. (A) is overlaid with a multislice image simulation based on ab initio calculations.

\section{References}

[1] Durnev et al. Phys. Rev. B. 2020, 101.12: 125420.

[2] Jones, L. Adv. Struct. Chem. Imaging 2015, 1 (1), 8.

[3] Pryor, A. et al.i Adv. Struct. Chem. Imaging 2017, 3 (1), 15. 